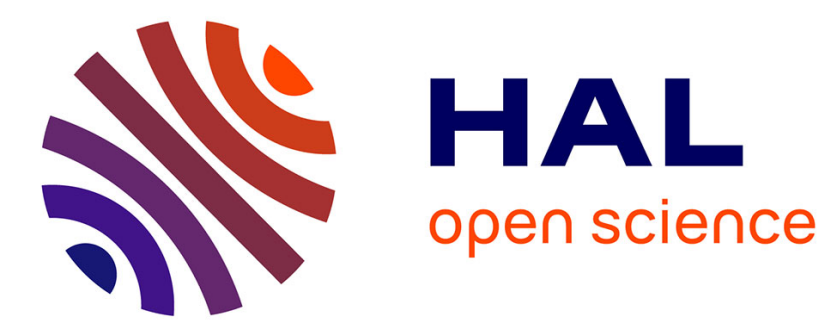

\title{
Multi-cell MIMO Transceiver Design for Mission-Critical Communication
}

Deepa Jagyasi, Alaa Daher, Marceau Coupechoux

\section{To cite this version:}

Deepa Jagyasi, Alaa Daher, Marceau Coupechoux. Multi-cell MIMO Transceiver Design for MissionCritical Communication. IEEE Globecom, Dec 2019, Honolulu, United States. hal-02315256

\author{
HAL Id: hal-02315256 \\ https://hal.science/hal-02315256
}

Submitted on 14 Oct 2019

HAL is a multi-disciplinary open access archive for the deposit and dissemination of scientific research documents, whether they are published or not. The documents may come from teaching and research institutions in France or abroad, or from public or private research centers.
L'archive ouverte pluridisciplinaire HAL, est destinée au dépôt et à la diffusion de documents scientifiques de niveau recherche, publiés ou non, émanant des établissements d'enseignement et de recherche français ou étrangers, des laboratoires publics ou privés. 


\title{
Multi-cell MIMO Transceiver design for Mission-Critical Communication
}

\author{
Deepa Jagyasi ${ }^{1}$, Marceau Coupechoux ${ }^{1}$ and Alaa Daher ${ }^{2}$ \\ ${ }^{1}$ INFRES, Telecom-ParisTech, University Paris-Saclay, France \\ \{deepa.jagyasi,marceau.coupechoux\}@ telecom-paristech.fr \\ ${ }^{2}$ ETELM, Les Ulis, France; alaa.daher@etelm.fr
}

\begin{abstract}
Business and Mission critical communication (MCC) is a major communication paradigm that is used by public agencies, e.g., during emergency situations, or critical infrastructure companies, e.g., airports, transportation, etc. MCC has very stringent requirements in terms of reliability, coverage and should offer group communications. Coordinated Multimedia Multicast/Broadcast single frequency network (MBSFN) is considered as a potential technology for MCC as it benefits from increased coverage and inter-cell interference mitigation. In this paper, we propose multi-input-multi-output (MIMO) multimedia MBSFN system design wherein each base station (BS) of a coordinated cluster multicasts a common message to all the users in a group. We use a greedy algorithm to dynamically form the cluster of synchronized BSs for optimal utilization of resources within an MBSFN. We assume the availability of perfect channel state information (CSI) knowledge and jointly obtain the optimal precoder and receive filters by minimizing the overall sum-meansquare-error (sum-MSE) constrained over the total transmit power. We further extend the proposed design to a robust case by considering the imperfections in available channel knowledge and obtain the transceiver matrices that are resilient to channel errors. We also present both the joint and robust system design for Single-Cell point-to-multipoint (SC-PTM) which is an alternative solution to MBSFN in MCC. Numerical results show the effectiveness of the proposed network architecture for future mission critical communication. Furthermore, the comparison results show that the proposed robust design demonstrate better performance and is resilient to the presence of CSI errors.

Keywords-mission-critical communication, dynamic clustering, MBSFN, SC-PTM, robust transceiver, imperfect CSI.
\end{abstract}

\section{INTRODUCTION}

Disaster relief teams require a reliable and efficient communication source during emergency circumstances like fire, ambulance services, natural calamities or terror attacks, known as mission critical communication (MCC). Business critical communications relate to communications between employees of critical infrastructures like airports, transportation networks, and energy operators, and have similar constraints as MCC. Two important requirements for MCC are indeed enhanced coverage and reliable group communications [1], [2]. In order to achieve these goals, coordinating MIMO-equipped BS transmissions within a cluster to serve a group of users in multicast is one of the standard approaches. In this paper, we thus propose a MIMO transceiver design for a cluster that allows both multicast and reliable communications for MCC. In the literature, several research efforts have been undertaken to design transceivers in a multicast scenario [3], [4]. For instance in [3], authors have proposed a linear precoder design over instantaneous rate maximization for multicasting system by using a cyclic alternating ascend based algorithm. A relayassisted MIMO precoder design for a multicast system have been proposed in [5]. Precoder for multi-group multicasting systems have been discussed in [6], [7]. However, all these works consider a single transmitting $\mathrm{BS}$, while in $\mathrm{MCC}$, clusters of BSs are coordinating to serve common data to all group users. Also as reliability is a major concern in MCC, transceiver designs considering rate-maximization optimization problem do not provide the best solution in MCC. Hence, we propose to design the MIMO transceivers by minimizing the sum-mean-square-error (sum-MSE) over all the group users in the network.

To further improve the robustness of the transmission, it is important to design schemes that are resilient to imperfect CSI estimation. Note that above references assume the availability of perfect CSI, while its estimation may be corrupted by various factors such as estimation error, feedback delays, quantization errors, pilot contamination, etc. Effect of imperfect CSI on the performance of MIMO systems is discussed in [8], which shows that transceivers that are designed assuming the availability of perfect CSI are not likely to achieve the desired performance in the presence of CSI errors. Robust design is addressed in the literature mostly for unicast or relayassisted communications, see e.g. [9], [10]. Only one reference is dealing with robust multicast [11]. In this paper, we extend these results to multiple clustered BSs in order to improve the reliability of MCC systems.

At system level, the way clustering of BSs is performed plays a crucial role on the system performance. There are two classical limit cases for clustering. On one hand, in MBSFN [12], all BSs of a large synchronization area participate to the transmission and use the same radio resources. On the other hand, in SC-PTM, introduced in Release 13 of 3GPP [13], each BS serves the group of users under its coverage area by using independent radio resources. MBSFN offers the best coverage at the cost of system capacity, while SC-PTM achieves the contrary [14]. Dynamic clustering offers a good trade-off to optimally utilize radio resources and achieve high reliability, as only the best BSs are activated to serve the group users [15], [16]. The performance of SCPTM and MBSFN has been discussed in literature and recent 3 GPP reports [17]. However, system level performance has not 


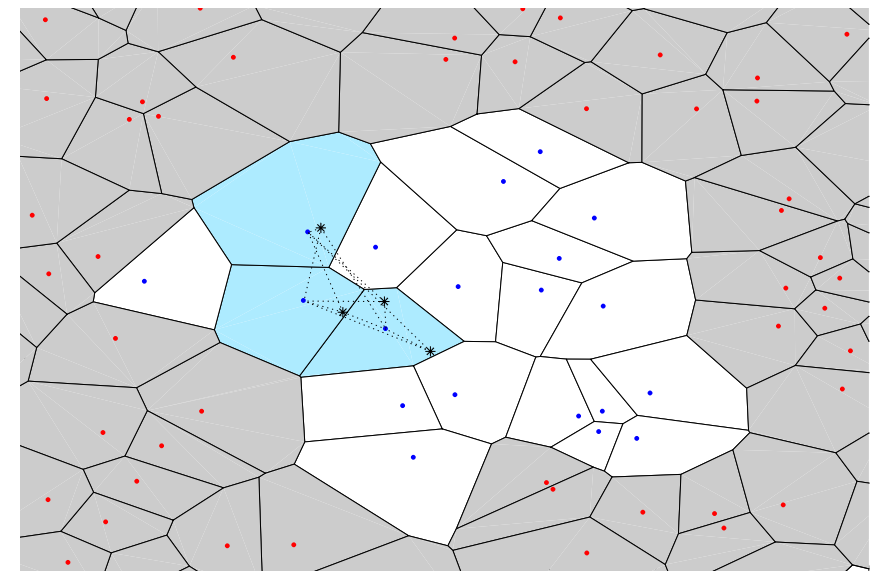

Fig. 1. Network model: White cells are part of MBSFN synchronization area Cell borders are represented using Voronoi tessellation. Black stars represent UE of a group. A group of users is served by a cluster of cells in blue of the synchronization area (connected with dotted lines).

been evaluated in conjunction with robust MIMO transceiver design.

The remainder of this paper is organized as follows. Section II describes the network and transceiver model. The proposed sum-MSE-based transceiver design for MCC is discussed first with perfect CSI in Section III-B and then with imperfect CSI in Section III-C. Section IV presents the simulation results and finally Section V concludes the paper. Notations: Throughout this paper, we use bold-faced lowercase letters to denote column vectors and bold-faced uppercase letters to denote matrices. For any matrix $\mathbf{X}, \operatorname{tr}(\mathbf{X}), \mathbb{E}\{\mathbf{X}\}$, $\mathbf{X}^{H}$, and $\mathbf{X}^{T}$ denote trace, expectation, conjugate transpose, and transpose operator, respectively.

\section{Network And Transceiver Model}

\section{A. Network Model}

We consider multi-user MIMO-downlink communication scenario with clustering to support group communication in MCC as shown in Fig. 1. There is a set of $\mathcal{B}$ BSs operating at the same frequency and serving groups of users. All these BSs are synchronized and form a MBSFN synchronization area (white cells in Fig. 1). All the (grey) cells outside this area are co-channel interferers. For a given group, a subset of BSs $\mathcal{C} \subseteq \mathcal{B}$ (blue cells) form a coordinated cluster to multicast a common data to all group users using the same time-frequency radio resources. In MBSFN, the cluster is formed by the whole synchronization area, i.e., $\mathcal{B}=\mathcal{C}$. In SC-PTM, all BSs use independent radio resources to serve the set of users located in their cell area using a multicast transmission, i.e., $|\mathcal{C}|=1$. All BSs thus interferes each others. In dynamic clustering, $\mathcal{C}$ is dynamically chosen for every group and is a subset of $\mathcal{B}$. In this paper, we adopt the greedy algorithm for dynamic clustering, which is well studied in the literature [15] and is detailed in Algorithm 1.

\section{B. Transceiver Model}

We focus here on the transmission between a set of $K_{1}$ BSs which form the cluster $\mathcal{C}$ to serve $K_{2}$ group users, see Fig. 2. Every BS in the cluster is equipped with $N_{T}$ transmit antennas

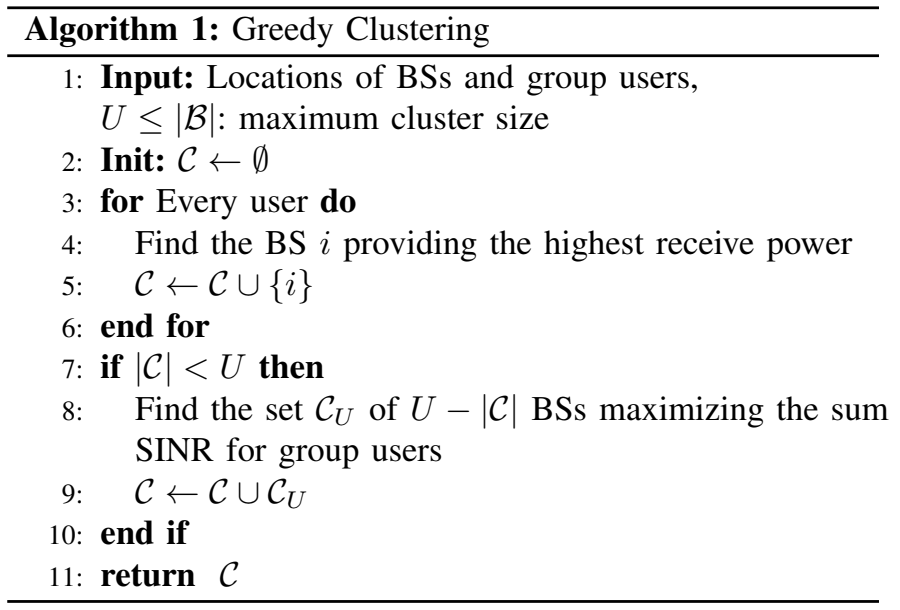

and user equipments (UEs) have $N_{R}$ receive antennas. Let $P_{i}$ be the total transmit power of the $i$-th BS. All the $K_{1}$ BSs multicast a common data sequence to be received by all the $K_{2}$ users. This data sequence is transmitted using $N_{s}$ parallel data streams. Let the signal transmitted by each BS in a single time slot be denoted by an $N_{s}$-dimensional column vector $\mathbf{d}$. Data symbols are uncorrelated so that $\mathbb{E}\left[\mathbf{d d}^{H}\right]=\mathbf{I}$. We assume linear transmit precoding at the BSs and linear receive filtering at the UEs. The precoded data $\mathbf{x}_{i}$ transmitted by $i$-th BS is given by $\mathbf{V}_{i} \mathbf{d}$, where $\mathbf{V}_{i}$ is the $N_{T} \times N_{s}$ precoder matrix at the $i$-th BS. Let the data received by the $k$-th UE be denoted by $\mathbf{y}_{k}$, which is further processed by a $N_{s} \times N_{R}$ RF beamformer $\mathbf{R}_{k}$ in order to obtain the desired estimate $\widehat{\mathbf{d}}_{k}$ of the transmitted data. The estimate of the transmitted data at user $k$ can now be given by:

$$
\begin{aligned}
\widehat{\mathbf{d}}_{k} & =\mathbf{R}_{k} \mathbf{y}_{k} \\
& =\mathbf{R}_{k}\left(\sum_{i=1}^{K_{1}} \alpha_{i k} \mathbf{C}_{i k} \mathbf{V}_{i} \mathbf{d}+\mathbf{n}_{k}\right),
\end{aligned}
$$

where $\mathbf{C}_{i k}$ and $\alpha_{i k}$ are the $N_{R} \times N_{T}$ channel gain due to fast fading and the distance dependent path-gain including shadowing between the $i$-th BS and the $k$-th user respectively, and $\mathbf{n}_{k}$ is the random noise vector at receiver $k$. We assume that the channel is modelled as a quasi-static Rayleigh fading channel, which is assumed to remain static over one time slot. Random noise is a circularly symmetric complex Gaussian white noise with zero mean and covariance matrix $\mathbb{E}\left[\mathbf{n}_{k} \mathbf{n}_{k}^{H}\right]=\sigma_{n_{k}}^{2} \mathbf{I}$. Noise and data are uncorrelated so that $\mathbb{E}\left[\mathbf{d n}_{k}^{H}\right]=0$.

\section{Transceiver Design for MCC}

\section{A. Problem Formulation}

In this section, we derive the precoder and receive filter matrices $\mathbf{V}_{i}$ and $\mathbf{R}_{k}$ by formulating a joint optimization problem that minimizes the sum mean-square-error under the constraint of each BS transmit power:

$$
\begin{array}{rc}
\min _{\left\{\mathbf{V}_{i}\right\},\left\{\mathbf{R}_{k}\right\}} & \sum_{k=1}^{K_{2}} \operatorname{MSE}_{k}=\sum_{k=1}^{K_{2}} \mathbb{E}\left\{\left\|\widehat{\mathbf{d}}_{k}-\mathbf{d}\right\|^{2}\right\}, \\
\text { s.t. } & \operatorname{tr}\left(\mathbf{V}_{i} \mathbf{V}_{i}^{H}\right) \leq P_{i}, i=1, \ldots, K_{1},
\end{array}
$$

where expectation is taken over data, channels, noise and estimation errors. Note that this problem is jointly non-convex in the $\mathbf{V}_{i}$ and $\mathbf{R}_{k}$ [18]. We look for a local optimum for this 


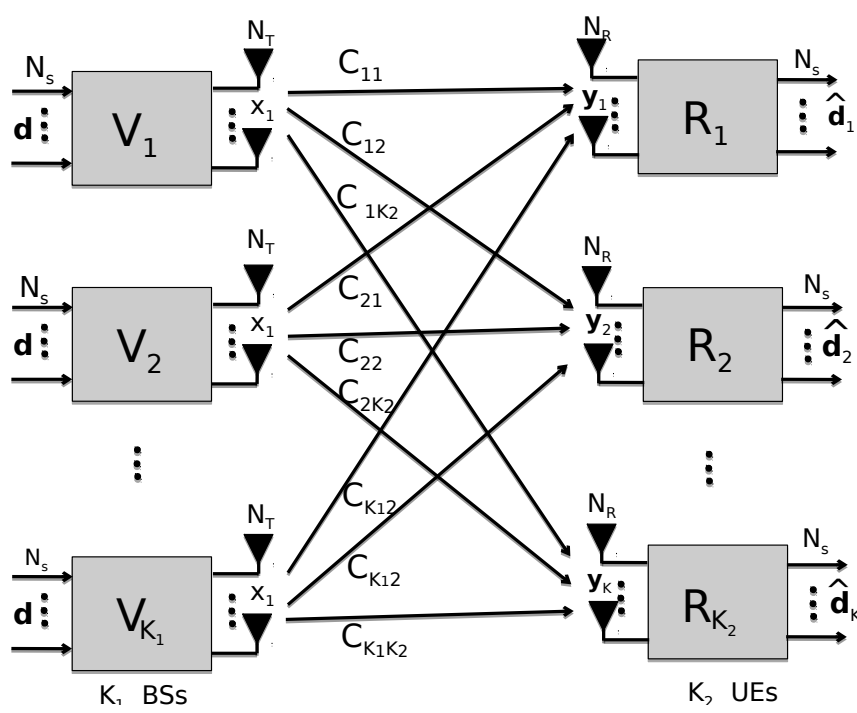

Fig. 2. MIMO-based transceiver model for $K_{1}$ BSs in a cluster serving a group of $K_{2}$ UEs in an MCC network.

problem by first assuming the availability of perfect CSI and then introduce estimation errors to obtain a robust transceiver design.

\section{B. Transceiver Design with Perfect CSI}

In this section, perfect CSI is assumed to be available at both the transmitters and receivers. This can possibly be achieved by exploiting the reciprocity of the channel or by feedback. The MSE at user $k$ can be given as:

$$
\begin{aligned}
\mathrm{MSE}_{k}= & \mathbb{E}\left\{\left\|\widehat{\mathbf{d}}_{k}-\mathbf{d}\right\|^{2}\right\} \\
= & \mathbb{E}\left[\operatorname{tr}\left(\mathbf{R}_{k} \sum_{i=1}^{K_{1}} \alpha_{i k} \mathbf{C}_{i k} \mathbf{V}_{i} \mathbf{d}+\mathbf{R}_{k} \mathbf{n}_{k}-\mathbf{d}\right)\right. \\
& \left.\left(\mathbf{R}_{k} \sum_{i=1}^{K} \alpha_{i k} \mathbf{C}_{i k} \mathbf{V}_{i} \mathbf{d}+\mathbf{R}_{k} \mathbf{n}_{k}-\mathbf{d}\right)^{H}\right] \\
= & \operatorname{tr}\left(\mathbf{R}_{k} \sum_{i=1}^{K_{1}} \alpha_{i k}^{2} \mathbf{C}_{i k} \mathbf{V}_{i} \mathbf{V}_{i}^{H} \mathbf{C}_{i k}^{H} \mathbf{R}_{k}^{H}+\sigma_{n_{k}}^{2} \mathbf{R}_{k} \mathbf{R}_{k}^{H}\right. \\
& \left.-\sum_{i=1}^{K_{1}} \alpha_{i k} \mathbf{V}_{i}^{H} \mathbf{C}_{i k}^{H} \mathbf{R}_{k}^{H}-\mathbf{R}_{k} \sum_{i=1}^{K_{1}} \alpha_{i k} \mathbf{C}_{i k} \mathbf{V}_{i}+\mathbf{I}\right) .
\end{aligned}
$$

Proposition 1: With perfect CSI, the optimal transmit precoders and receive filters verify the following equations:

$$
\begin{array}{r}
\mathbf{V}_{i}=\left[\sum_{k=1}^{K_{2}} \alpha_{i k}^{2} \mathbf{C}_{i k}^{H} \mathbf{R}_{k}^{H} \mathbf{R}_{k} \mathbf{C}_{i k}+\lambda_{i} \mathbf{I}\right]^{-1} \sum_{k=1}^{K_{2}} \alpha_{i k} \mathbf{C}_{i k}^{H} \mathbf{R}_{k}^{H},(5) \\
\mathbf{R}_{k}=\sum_{i=1}^{K_{1}} \alpha_{i k} \mathbf{V}_{i}^{H} \mathbf{C}_{i k}^{H}\left[\sum_{i=1}^{K_{1}} \alpha_{i k}^{2} \mathbf{C}_{i k} \mathbf{V}_{i} \mathbf{V}_{i}^{H} \mathbf{C}_{i k}^{H}+\sigma_{n_{k}}^{2} \mathbf{I}\right]^{-1}(6)
\end{array}
$$

where $\lambda_{i} \geq 0$ is a Lagrangian multiplier, which should be chosen so that:

$$
\operatorname{tr}\left(\mathbf{V}_{i}\left(\lambda_{i}\right)^{H} \mathbf{V}_{i}\left(\lambda_{i}\right)\right)=P_{i}
$$

Proof: See Appendix A, with $\chi=0$.

It can be observed that equations (5) and (6) are interdependent and also depend on the Lagrange multipliers.
Hence, we use an iterative coordinate descent method to obtain the values of each variable as given in Algorithm 2 .

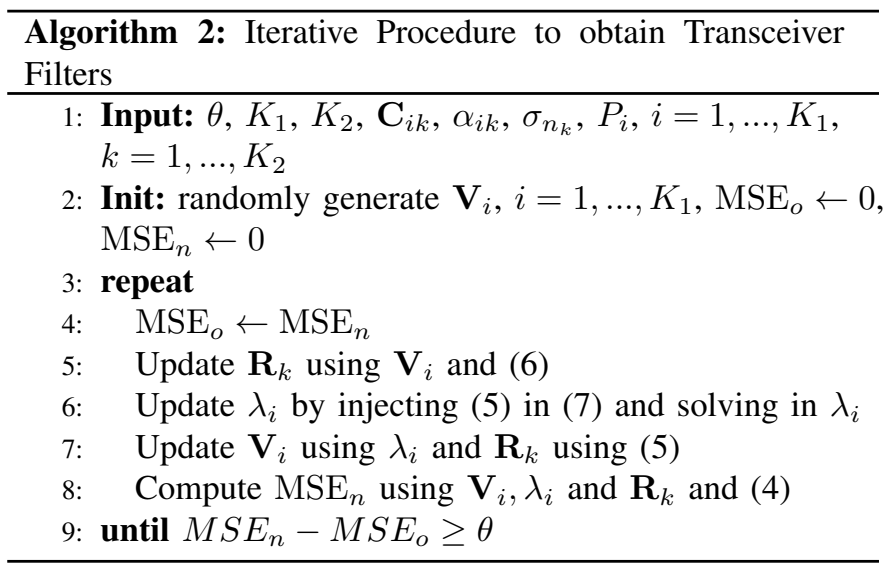

\section{Robust Transceiver Design}

In order to mitigate the effect of CSI errors, we propose a robust transceiver design by considering the CSI errors in the channel, assuming that these errors are stochastically distributed Gaussian random variables with known error variance. We incorporate CSI error in the optimization problem to obtain a robust design. Let the CSI error variance be $\sigma_{E}^{2}$. Thus, the channel for the robust transceiver design can be given as:

$$
\mathbf{C}=\widehat{\mathbf{C}}+\boldsymbol{\Delta} \text {, }
$$

where $\widehat{\mathbf{C}}$ is the estimated channel and $\boldsymbol{\Delta} \sim \mathcal{N}\left(0, \sigma_{E}^{2}\right)$. The received signal at user $k$ is now given as:

$$
\mathbf{y}_{k}=\sum_{i=1}^{K_{1}} \alpha_{i k}\left(\widehat{\mathbf{C}}_{i k}+\boldsymbol{\Delta}_{i k}\right) \mathbf{V}_{i} \mathbf{d}+\mathbf{n}_{k}
$$

and the estimate of transmitted data at $k$-th receiver is:

$$
\widehat{\mathbf{d}}_{k}=\mathbf{R}_{k} \sum_{k=1}^{K_{1}} \alpha_{i k}\left(\widehat{\mathbf{C}}_{i k}+\boldsymbol{\Delta}_{i k}\right) \mathbf{V}_{i} \mathbf{d}+\mathbf{R}_{k} \mathbf{n}_{k} .
$$

Hence, the MSE for $k$-th user can be written:

$$
\begin{aligned}
\mathrm{MSE}_{k}= & \mathbb{E}\left\{\left\|\widehat{\mathbf{d}}_{k}-\mathbf{d}\right\|^{2}\right\} \\
= & \mathbb{E}\left[\operatorname{tr}\left(\mathbf{R}_{k} \sum_{i=1}^{K_{1}} \alpha_{i k}\left(\widehat{\mathbf{C}}_{i k}+\boldsymbol{\Delta}_{i k}\right) \mathbf{V}_{i} \mathbf{d}+\mathbf{R}_{k} \mathbf{n}_{k}-\mathbf{d}\right)\right. \\
& \left.\left(\mathbf{R}_{k} \sum_{i=1}^{K_{1}} \alpha_{i k}\left(\widehat{\mathbf{C}}_{i k}+\boldsymbol{\Delta}_{i k}\right) \mathbf{V}_{i} \mathbf{d}+\mathbf{R}_{k} \mathbf{n}_{k}-\mathbf{d}\right)^{H}\right] \\
= & \operatorname{tr}\left(\mathbf{R}_{k} \sum_{i=1}^{K_{1}} \alpha_{i k}^{2}\left(\widehat{\mathbf{C}}_{i k} \mathbf{V}_{i} \mathbf{V}_{i}^{H} \widehat{\mathbf{C}}_{i k}^{H}\right) \mathbf{R}_{k}^{H}+\sigma_{n_{k}}^{2} \mathbf{R}_{k} \mathbf{R}_{k}^{H}\right. \\
& \left.-\sum_{i=1}^{K_{1}} \alpha_{i k} \mathbf{V}_{i}^{H} \widehat{\mathbf{C}}_{i k}^{H} \mathbf{R}_{k}^{H}-\mathbf{R}_{k} \sum_{i=1}^{K_{1}} \alpha_{i k} \widehat{\mathbf{C}}_{i k} \mathbf{V}_{i}+\mathbf{I}\right)+ \\
& \mathbb{E}\left[\operatorname{tr}\left(\mathbf{R}_{k} \sum_{i=1}^{K_{1}} \alpha_{i k}^{2} \boldsymbol{\Delta}_{i k} \mathbf{V}_{i} \mathbf{V}_{i}^{H} \boldsymbol{\Delta}_{i k}^{H} \mathbf{R}_{k}^{H}\right)\right] .
\end{aligned}
$$

Now, for any matrix $\mathbf{X}$ with $\mathbb{E}\left\{\mathbf{X X}^{H}\right\}=\sigma^{2} \mathbf{I}$, and matrices $\mathbf{U}$ and $\mathbf{V}$ of appropriate dimensions, the following equality holds [9]:

$$
\mathbb{E}\left[\operatorname{tr}\left(\mathbf{X} \mathbf{U} \mathbf{X}^{H} \mathbf{V}\right)\right]=\mathbb{E}\left[\operatorname{tr}\left(\mathbf{X}^{H} \mathbf{V X U}\right)\right]=\sigma^{2} \operatorname{tr}(\mathbf{U}) \operatorname{tr}(\mathbf{V}) .
$$




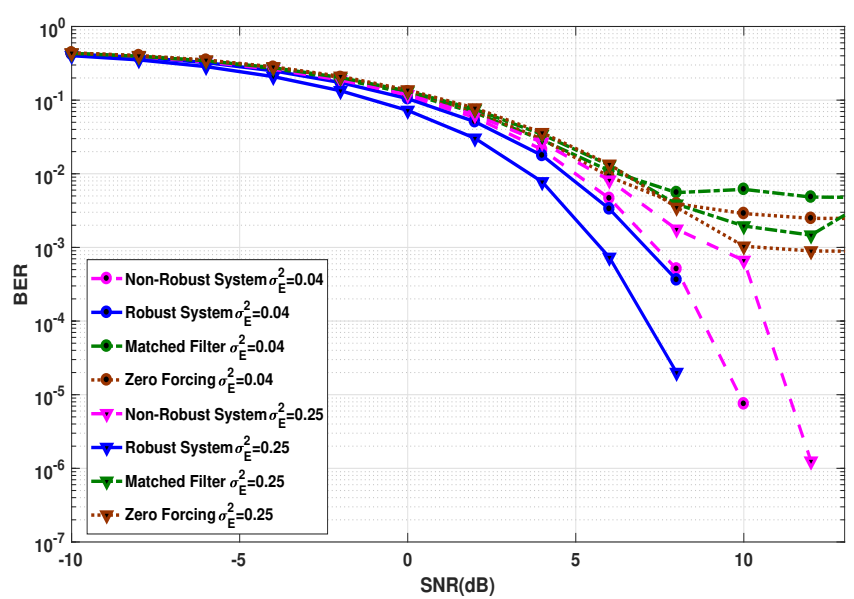

Fig. 3. BER Vs SNR in $\mathrm{dB}$ for different transceiver schemes.

Incorporating this result into (11), the robust MSE at user $k$ can be given as:

$$
\begin{aligned}
\mathrm{MSE}_{k}= & \operatorname{tr}\left(\mathbf{R}_{k} \sum_{i=1}^{K_{1}} \alpha_{i k}^{2} \widehat{\mathbf{C}}_{i k} \mathbf{V}_{i} \mathbf{V}_{i}^{H} \widehat{\mathbf{C}}_{i k}^{H} \mathbf{R}_{k}^{H}+\sigma_{n_{k}}^{2} \mathbf{R}_{k} \mathbf{R}_{k}^{H}-\right. \\
& \left.\sum_{i=1}^{K_{1}} \alpha_{i k} \mathbf{V}_{i}^{H} \widehat{\mathbf{C}}_{i k}^{H} \mathbf{R}_{k}^{H}-\mathbf{R}_{k} \sum_{i=1}^{K_{1}} \alpha_{i k} \widehat{\mathbf{C}}_{i k} \mathbf{V}_{i}+\mathbf{I}\right)+ \\
& \sum_{i=1}^{K_{1}} \alpha_{i k}^{2} \sigma_{E i k}^{2} \operatorname{tr}\left(\mathbf{R}_{j} \mathbf{R}_{j}^{H}\right) \operatorname{tr}\left(\mathbf{V}_{i} \mathbf{V}_{i}^{H}\right) .
\end{aligned}
$$

We consider the similar optimization problem as in previous section, i.e., (2) and (3).

Proposition 2: In presence of Gaussian errors on channel estimation, the optimal transmit precoders and receive filters verify the following equations:

$$
\begin{aligned}
\mathbf{V}_{i}= & {\left[\sum_{k=1}^{K_{2}} \alpha_{i k}^{2} \widehat{\mathbf{C}}_{i k}^{H} \mathbf{R}_{k}^{H} \mathbf{R}_{k} \widehat{\mathbf{C}}_{i k}+\sum_{k=1}^{K_{2}} \alpha_{i k}^{2} \sigma_{E i k}^{2} \operatorname{tr}\left(\mathbf{R}_{k}^{H} \mathbf{R}_{k}\right) \mathbf{I}\right.} \\
& \left.+\lambda_{i} \mathbf{I}\right]^{-1} \sum_{i=1}^{K 2} \alpha_{i k} \widehat{\mathbf{C}}_{i k}^{H} \mathbf{R}_{k}^{H}, \\
\mathbf{R}_{k}= & \sum_{i=1}^{K_{1}} \alpha_{i k} \mathbf{V}_{i}^{H} \widehat{\mathbf{C}}_{i k}^{H}\left[\sum_{i=1}^{K_{1}} \alpha_{i k}^{2} \widehat{\mathbf{C}}_{i k} \mathbf{V}_{i} \mathbf{V}_{i}^{H} \widehat{\mathbf{C}}_{i k}^{H}+\sum_{i=1}^{K_{1}} \alpha_{i k}^{2} \sigma_{E i k}^{2}\right. \\
& \left.\operatorname{tr}\left(\mathbf{V}_{i} \mathbf{V}_{i}^{H}\right) \mathbf{I}+\sigma_{n_{k}}^{2} \mathbf{I}\right]^{-1} .
\end{aligned}
$$

where $\lambda_{i} \geq 0$ is a Lagrangian multiplier, which should be chosen so that:

$$
\operatorname{tr}\left(\mathbf{V}_{i}\left(\lambda_{i}\right)^{H} \mathbf{V}_{i}\left(\lambda_{i}\right)\right)=P_{i}
$$

Proof: See Appendix A, with $\chi=1$.

Again, we can find the precoders and filter matrices using the same iterative approach given in Algorithm 2.

\section{Simulation Results}

In this section, we illustrate the performance of the proposed MCC system design. We first study the physical layer performance and then perform system level simulations.

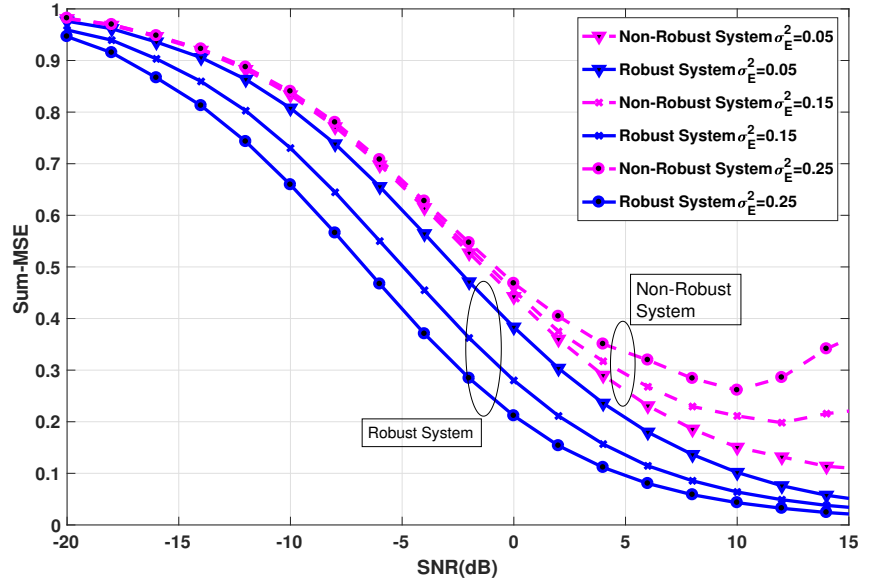

Fig. 4. Sum-MSE Vs SNR in $\mathrm{dB}$ for the proposed non-robust and robust transceiver schemes with different channel error variances.

\section{A. Physical Layer Simulation}

We compare the performance of proposed robust and nonrobust sum-MSE-based transceiver designs in terms of BER and sum-MSE, averaged over all the UEs. We consider $K_{1}=4$ BSs serving $K_{2}=16$ group users. The transmit power associated with each $\mathrm{BS}$ is assumed to be $P_{i}=46 \mathrm{dBm}$, $i=1,2, . ., K_{1}$ and noise variance at all the receiving nodes is set to $\sigma_{n_{k}}^{2}=P_{i} / \mathrm{SNR}, k=1,2, . ., K_{2}$, where $\mathrm{SNR}$ is varied in simulations. QPSK modulation scheme is assumed for generation of data and all the simulations have been tested for $N=10^{6}$ data samples. The convergence threshold $\theta$ considered for the proposed iterative algorithm in Algorithm 2 is $10^{-4}$. In order to incorporate the robustness in the system design, we consider the channel estimation errors variance to be $\sigma_{E}^{2} \in\{0.04,0.05,0.15,0.25\}$ during the simulation.

In Fig. 3, we evaluate the BER versus SNR for different values of $\sigma_{E}^{2}$ while considering $N_{T}=8, N_{R}=4, N_{s}=2$, $\sigma_{E}^{2} \in\{0.04,0.25\}$ for proposed optimal robust and non-robust transceiver designs in comparison with classical zero forcing and matched filter receiver. It is observed that the proposed schemes achieve lower BER as compared to classical schemes for complete range of SNR. Moreover, the proposed robust scheme demonstrates better performance than that of the nonrobust scheme. With simulation parameters set as $N_{T}=8$, $N_{R}=4, N_{s}=2, \sigma_{E}^{2} \in\{0.05,0.15,0.25\}$ in Fig. 4, we compare the proposed transceiver designs in terms of average sum-MSE performance versus SNR in the presence of CSI errors and observe that the robust design achieves lower sumMSE as compared to the non-robust design. It is seen that the difference between the performance of both the designs increases with increasing error variance. Hence we observe the robustness of the proposed robust design towards CSI errors. Fig. 5 depicts the effect of varying transmit antennas in terms of BER performance for the proposed robust and non-robust transceiver designs for $N_{R}=4, N_{s}=2$, and $\sigma_{E}^{2}=0.04$. It is observed that the system performance improves with increasing number of transmit antenna's, hence making it amenable for MIMO implementation. 


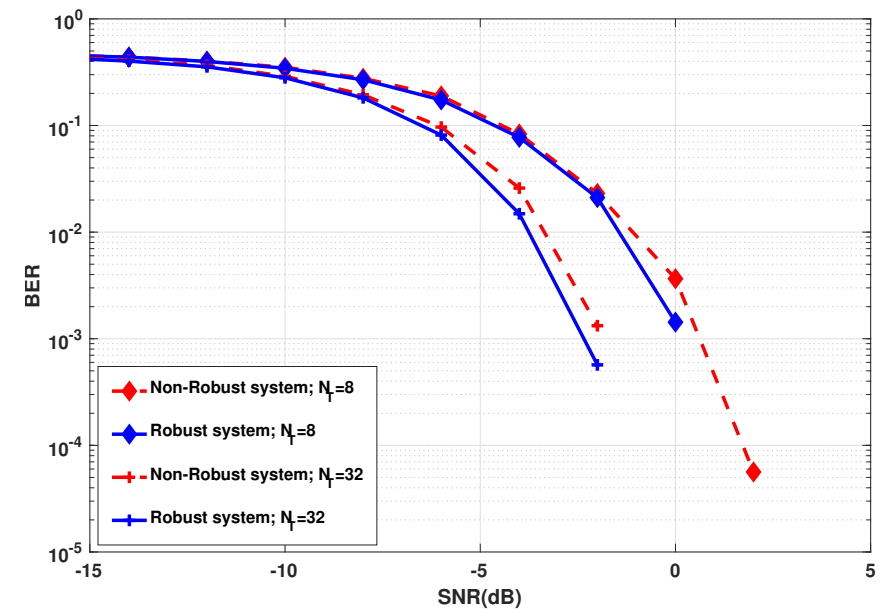

Fig. 5. BER Vs SNR in $\mathrm{dB}$ for robust and non-robust system with different numbers of transmit antennas.

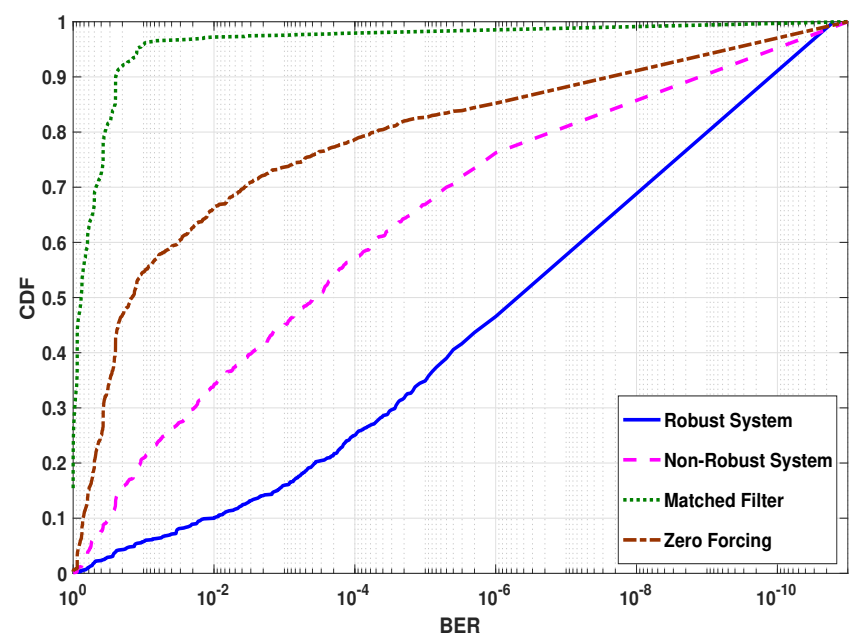

Fig. 6. CDF of the BER for mateched filter, zero forcing, non-robust and robust optimal designs.

\section{B. System Level Simulation}

In this subsection, we simulate our transceiver design at system level. We consider $100 \mathrm{BSs}$ which are distributed over an area of $10 \mathrm{~km}^{2}$, drawn according to a Poisson process. The synchronization area is made of $20 \mathrm{BSs}$ (see Fig. 1). Cell edges are obtained from the Voronoi tessellation. We assume $10 \mathrm{UEs}$ per cell which are also distributed according to a Poisson process. The path loss between BSs and UEs is calculated as per Okumura-Hata model using a carrier frequency of $700 \mathrm{MHz}$ as given in [19] (a typical frequency for MCC). The noise power considered at the UEs is assumed to have both thermal noise and co-channel interference from other BSs outside the cluster. Throughout the simulations, we consider $N_{T}=16, N_{R}=8, N_{s}=2, \sigma_{E}^{2}=0.1$ and $P_{i}=46 \mathrm{dBm}$, $i=1,2, . ., K_{1}$. We select $K_{2}=10$ group users by first choosing uniformly random in the synchronization area a team leader for the group and afterwards select the remaining group users within its distance range of $500 \mathrm{~m}$ uniformly random. All the simulations are executed over $10^{6}$ data streams.

In Fig. 6 we show the Cumulative Distribution Function

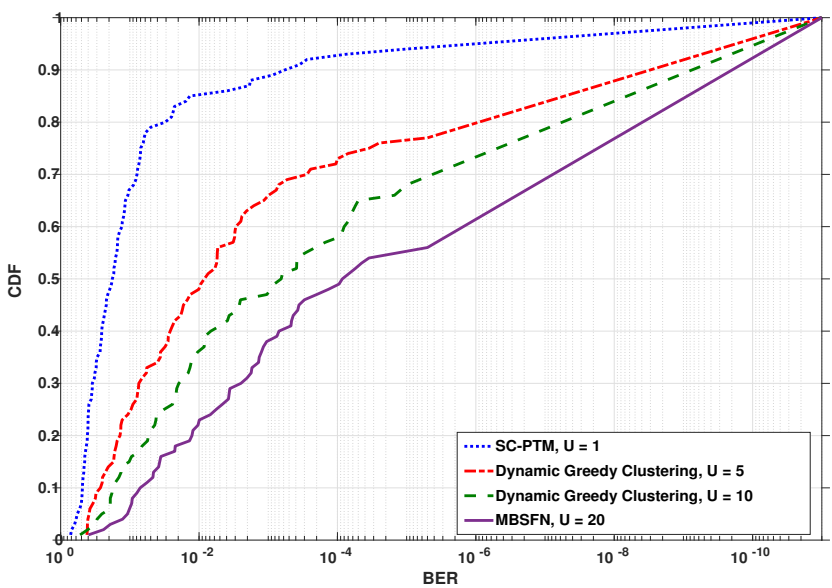

Fig. 7. CDF of the BER for MBSFN, SC-PTM and dynamic greddy clustering.

(CDF) of the BER at every user for different transceiver schemes where the CDF is obtained by averaging over 100 different groups for all the group users. It is observed that the proposed robust scheme achieves significantly lower BER as compared to all other schemes. For instance at BER threshold of $10^{-2}$, the performance of matched filter saturates with more than $90 \%$ of users having higher than $10^{-2}$ BER, while in case of zero forcing receiver about $68 \%$ of users do not meet the threshold. The proposed designs are observed to perform much better with only $35 \%$ and $10 \%$ users below the desired threshold in case of non-robust and robust transceiver designs respectively. In Fig. 7, we compare the CDF of the BER for MBSFN (equivalent to dynamic greedy clustering with $U=20)$, SC-PTM $(U=1)$ and dynamic greedy clustering with $U \in\{5,10\}$ with the proposed robust transceiver scheme. We see that the more BSs are involved in the transmission, the better is the performance. Higher cluster sizes imply however less capacity for the whole system. In terms of coverage, MBSFN thus performs the best, SC-PTM the worst and dynamic greedy clustering is a trade-off.

\section{CONCLUSION}

In this paper, we propose a MIMO transceiver design for MBSFN, SC-PTM, and dynamic clustering, serving group of users in a MCC scenario. A sum-MSE minimizing optimization problem is formulated under a constraint on total transmit power for the precoder and receive filter design. We assume the availability of perfect CSI knowledge at all the coordinated BSs and UEs in a group and obtain the optimal transceiver matrices. We adopt a greedy algorithm to obtain a cluster of coordinated BSs which operate in synchronization with each other and serve users in a group, hence reducing the overall resource utilization in MCC. We also propose a robust system design by assuming imperfect CSI. We model the errors in the available CSI as stochastic errors with known error variance where probability distribution of the CSI error is Gaussian. We compare the performance of all the proposed designs for different values of system parameters in MCC scenario. Simulation results illustrate that the proposed system design 
outperforms the existing designs in literature. Furthermore, the proposed robust system demonstrates improved performance over non-robust design in the presence of CSI errors.

\section{APPENDIX A}

\section{PROOF FOR PROPOSITION 1 AND PROPOSITION 2}

In this section we present a generalized solution for both the proposed schemes as discussed in Section III-B and III-C. We reformulate the optimization problem defined in (2) and (3) as a generalized Lagrangian solution L by introducing a binary slack variable $\chi$ to maintain the generality between both the propositions. We consider $\chi=1$ for robust solution and $\chi=0$ otherwise. Thus the generalized Lagrangian $\mathrm{L}$ for the optimization problem is given as:

$$
\begin{aligned}
& \mathrm{L}\left(\mathbf{V}_{i}, \mathbf{R}_{k}, \lambda_{i}\right)=\sum_{k=1}^{K_{2}} \mathrm{MSE}_{\mathrm{k}}+\sum_{i=1}^{K_{1}} \lambda_{i}\left(\operatorname{tr}\left(\mathbf{V}_{i}^{H} \mathbf{V}_{i}\right)-P_{i}\right) \\
& =\sum_{k=1}^{K_{2}}\left(\operatorname { t r } \left(\mathbf{R}_{k} \sum_{i=1}^{K_{1}} \alpha_{i k}^{2} \widehat{\mathbf{C}}_{i k} \mathbf{V} i \mathbf{V}_{i}^{H} \widehat{\mathbf{C}}_{i k}^{H} \mathbf{R}_{k}^{H}+\sigma_{n_{k}}^{2} \mathbf{R}_{k} \mathbf{R}_{k}^{H}-\right.\right. \\
& \left.\sum_{i=1}^{K_{1}} \alpha_{i k} \mathbf{V}_{i}^{H} \mathbf{C}_{i k}^{H} \mathbf{R}_{k}^{H}\right)-\mathbf{R}_{k} \sum_{i=1}^{K_{1}} \alpha_{i k} \mathbf{C}_{i k} \mathbf{V}_{i}+\mathbf{I}+\chi \sum_{i=1}^{K_{1}} \alpha_{i k}^{2} \sigma_{E i k}^{2} \\
& \left.\operatorname{tr}\left(\mathbf{R}_{k} \mathbf{R}_{k}^{H}\right) \operatorname{tr}\left(\mathbf{V}_{i} \mathbf{V}_{i}^{H}\right)\right)+\sum_{i=1}^{K_{1}} \lambda_{i}\left(\operatorname{tr}\left(\mathbf{V}_{i}^{H} \mathbf{V}_{i}\right)-P_{i}\right),
\end{aligned}
$$

We obtain the desired precoder and receive filter matrices by minimizing the Lagrangian with respect to each dependent variable. We first minimize the Lagrangian for $\left\{\mathbf{V}_{i}\right\}$ while keeping $\left\{\mathbf{R}_{k}\right\}$ and $\left\{\lambda_{i}\right\}$ fixed. Thus, by taking the zerogradient of (17) w.r.t. $\mathbf{V}_{i}^{H}$ we get:

$$
\begin{aligned}
& \frac{\partial L}{\partial \mathbf{V}_{i}^{H}}=\sum_{k=1}^{K_{2}} \alpha_{i k}^{2} \widehat{\mathbf{C}}_{i k}^{H} \mathbf{R}_{k}^{H} \mathbf{R}_{k} \widehat{\mathbf{C}}_{i k} \mathbf{V}_{i}-\sum_{k=1}^{K_{2}} \alpha_{i k} \widehat{\mathbf{C}}_{i k}^{H} \mathbf{R}_{k}^{H}+ \\
& \chi \sum_{k=1}^{K_{2}} \alpha_{i k}^{2} \sigma_{E i k}^{2} \operatorname{tr}\left(\mathbf{R}_{k} \mathbf{R}_{k}^{H}\right) \mathbf{V}_{i}+\lambda_{i} \mathbf{V}_{i}=0, \\
& \Longrightarrow \mathbf{V}_{i}=\left(\sum_{k=1}^{K_{2}} \alpha_{i k}^{2} \widehat{\mathbf{C}}_{i k}^{H} \mathbf{R}_{k}^{H} \mathbf{R}_{k} \widehat{\mathbf{C}}_{i k}+\chi \sum_{k=1}^{K_{2}} \alpha_{i k}^{2} \sigma_{E i k}^{2}\right. \\
& \left.\operatorname{tr}\left(\mathbf{R}_{k}^{H} \mathbf{R}_{k}\right) \mathbf{I}+\lambda_{i} \mathbf{I}\right)^{-1} \sum_{k=1}^{K_{2}} \alpha_{i k} \widehat{\mathbf{C}}_{i k}^{H} \mathbf{R}_{k}^{H} .
\end{aligned}
$$

In the same way, we differentiate the Lagrangian with respect to $\mathbf{R}_{k}^{H}$ while keeping $\left\{\mathbf{V}_{i}\right\}$ and $\left\{\lambda_{i}\right\}$ fixed and setting it to zero, we get:

$$
\begin{aligned}
\frac{\partial L}{\partial \mathbf{R}_{k}^{H}}= & \mathbf{R}_{k} \sum_{i=1}^{K_{1}} \alpha_{i k}^{2} \widehat{\mathbf{C}}_{i k} \mathbf{V} i \mathbf{V}_{i}^{H} \widehat{\mathbf{C}}_{i k}^{H}-\sum_{i=1}^{K_{1}} \alpha_{i k} \mathbf{V}_{i}^{H} \mathbf{C}_{i k}^{H}+ \\
& \sigma_{n_{k}}^{2} \mathbf{R}_{k}+\chi \sum_{i=1}^{K_{1}} \alpha_{i k}^{2} \sigma_{E i k}^{2} \operatorname{tr}\left(\mathbf{V}_{i} \mathbf{V}_{i}^{H}\right) \mathbf{R}_{k}=0 .
\end{aligned}
$$

By solving, we have:

$$
\begin{aligned}
\mathbf{R}_{k}= & \sum_{i=1}^{K_{1}} \alpha_{i k} \mathbf{V}_{i}^{H} \widehat{\mathbf{C}}_{i k}^{H}\left[\sum_{i=1}^{K_{1}} \alpha_{i k}^{2} \widehat{\mathbf{C}}_{i k} \mathbf{V}_{i} \mathbf{V}_{i}^{H} \widehat{\mathbf{C}}_{i k}^{H}+\right. \\
& \left.\chi \sum_{i=1}^{K_{1}} \alpha_{i k}^{2} \sigma_{E i k}^{2} \operatorname{tr}\left(\mathbf{V}_{i} \mathbf{V}_{i}^{H}\right) \mathbf{I}+\sigma_{n_{k}}^{2} \mathbf{I}\right]^{-1} .
\end{aligned}
$$

We thus obtain a set of necessary conditions for optimality.

\section{REFERENCES}

[1] S. W. Choi, Y. Song, W. Shin, and J. Kim, "A feasibility study on mission-critical push-to-talk: Standards and implementation perspectives," IEEE Communications Magazine, vol. 57, no. 2, pp. 81-87, February 2019.

[2] O. Vikhrova, S. Pizzi, A. Molinaro, K. Samouylov, and G. Araniti, "Group-oriented services for critical machine type communications in 5G networks," in Proc. IEEE 29th Annual International Symposium on Personal, Indoor and Mobile Radio Communications (PIMRC), Sep. 2018, pp. 824-828.

[3] H. Zhu, N. Prasad, and S. Rangarajan, "Precoder design for physical layer multicasting," IEEE Transactions on Signal Processing, vol. 60, no. 11, pp. 5932-5947, Nov 2012.

[4] E. Chiu and V. K. N. Lau, "Precoding design for multi-antenna multicast broadcast services with limited feedback," IEEE Systems Journal, vol. 4, no. 4, pp. 550-560, Dec 2010.

[5] M. R. A. Khandaker and Y. Rong, "Precoding design for MIMO relay multicasting," IEEE Transactions on Wireless Communications, vol. 12, no. 7, pp. 3544-3555, July 2013.

[6] M. Sadeghi, E. Bjoernson, E. G. Larsson, C. Yuen, and T. L. Marzetta, "Multigroup multicast precoding in massive mimo," in Proc. IEEE Global Communications Conference (GLOBECOM), Dec 2017, pp. 1-6.

[7] C. Lee and W. Chung, "Max-min hybrid precoding in millimeter wave cooperative MISO systems," in Proc. IEEE International Conference on Communications (ICC), May 2016, pp. 1-6.

[8] N. Lee, O. Simeone, and J. Kang, "The effect of imperfect channel knowledge on a MIMO system with interference," IEEE Transactions on Communications, vol. 60, no. 8, pp. 2221-2229, August 2012.

[9] P. Ubaidulla and A. Chockalingam, "Relay precoder optimization in MIMO-relay networks with imperfect CSI," IEEE Transactions on Signal Processing, vol. 59, no. 11, pp. 5473-5484, Nov 2011.

[10] L. Gopal, Y. Rong, and Z. Zang, "Robust MMSE transceiver design for nonregenerative multicasting MIMO relay systems," IEEE Transactions on Vehicular Technology, vol. 66, no. 10, pp. 8979-8989, Oct 2017.

[11] P. Ubaidulla and A. Chockalingam, "Precoder designs for MIMO broadcast channels with imperfect CSI," in Proc. IEEE International Conference on Wireless and Mobile Computing, Networking and Communications, Oct 2008, pp. 145-150.

[12] A. Daher, M. Coupechoux, P. Godlewski, J. Kelif, P. Ngouat, and P. Minot, "SINR model for MBSFN based mission critical communications," in Proc. IEEE 86th Vehicular Technology Conference (VTC-Fall), Sep. 2017, pp. $1-5$

[13] MCC Work Plan Manager (Alain Sultan), "Release 13 analytical view version sept. 9th 2015," 3rd Generation Patnership Project (3GPP), Tech. Rep. TSG RP-151569, Sept 2015.

[14] Huawei, HiSilicon, TD-Tech, SouthernLINC, Potevio, China Unicom, MediaTek Inc., CATT, "Comparison of SC-PTM and MBSFN use for public safety," 3rd Generation Patnership Project (3GPP), Tech. Rep. TSG RP-151569, Apr 2015.

[15] A. Papadogiannis, D. Gesbert, and E. Hardouin, "A dynamic clustering approach in wireless networks with multi-cell cooperative processing," in Proc. IEEE International Conference on Communications, May 2008, pp. 4033-4037.

[16] M. R. Aktar, M. F. Hossain, and M. Al-Hasan, "Dynamic clustering approach for interference cancellation in downlink c-ran," in Proc. International Conference on Computer, Communication, Chemical, Material and Electronic Engineering (IC4ME2), Feb 2018, pp. 1-4.

[17] A. Daher, M. Coupechoux, P. Godlewski, P. Ngouat, and P. Minot, "SCPTM or MBSFN for mission critical communications?" in Proc. IEEE 85th Vehicular Technology Conference (VTC Spring), June 2017, pp. $1-6$.

[18] F. Wang, X. Yuan, S. C. Liew, and D. Guo, "Wireless MIMO switching: Weighted sum mean square error and sum rate optimization," IEEE Transactions on Information Theory, vol. 59, no. 9, pp. 5297-5312, Sep. 2013.

[19] M. Hata, "Empirical formula for propagation loss in land mobile radio services," IEEE Transactions on Vehicular Technology, vol. 29, no. 3, pp. 317-325, Aug 1980. 